\title{
Self-management interventions for type 2 diabetes in adult people with severe mental illness (Protocol)
}

\author{
McBain H, Mulligan K, Haddad M, Flood C, Jones J, Simpson A
}

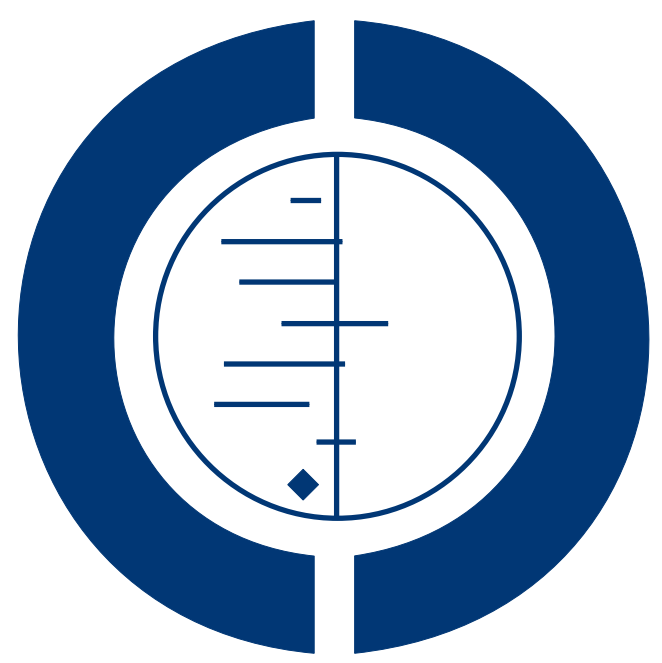

\section{THE COCHRANE COLLABORATION $^{\circledR}$}

This is a reprint of a Cochrane protocol, prepared and maintained by The Cochrane Collaboration and published in The Cochrane Library 2014, Issue 11

http://www.thecochranelibrary.com

\section{WILEY}


TABLE OF CONTENTS

HEADER . . . . . . . . . . . . . . . . . . . . . . . . . . . . . . . . . . 1

ABSTRACT . . . . . . . . . . . . . . . . . . . . . . . . . . . . . . . . . . . . . . . 1

BACKGROUND . . . . . . . . . . . . . . . . . . . . . . . . . . . . . . . . . . . . . . . . . . . . . .

Figure 1. . . . . . . . . . . . . . . . . . . . . . . . . . . . . . . . . . . . . . 4

OBJECTIVES . . . . . . . . . . . . . . . . . . . . . . . . . . . . . . . . . . . . . . .

METHODS . . . . . . . . . . . . . . . . . . . . . . . . . . . . . . . . . . . . . . 5

ACKNOWLEDGEMENTS . . . . . . . . . . . . . . . . . . . . . . . . . . . . . . . . . . . . . . .

REFERENCES . . . . . . . . . . . . . . . . . . . . . . . . . . . . . . . . . . . . . 10

APPENDICES . . . . . . . . . . . . . . . . . . . . . . . . . . . . . . . . . . . . . 12

CONTRIBUTIONS OF AUTHORS . . . . . . . . . . . . . . . . . . . . . . . . . . . . . . . . . . . . . . .

DECLARATIONS OF INTEREST . . . . . . . . . . . . . . . . . . . . . . . . . . . . . . . . . . . . .

NOTES . . . . . . . . . . . . . . . . . . . . . . . . . . . . . . . . . . . . . . . . 23 


\title{
Self-management interventions for type $\mathbf{2}$ diabetes in adult people with severe mental illness
}

\author{
Hayley McBain ${ }^{1}$, Kathleen Mulligan $^{1}$, Mark Haddad $^{1}$, Chris Flood $^{1}$, Julia Jones ${ }^{1}$, Alan Simpson ${ }^{1}$ \\ ${ }^{1}$ School of Health Sciences, City University London, London, UK \\ Contact address: Hayley McBain, School of Health Sciences, City University London, Northampton Square, London, EC1V 0HB, \\ UK. hayley.mcbain.1@city.ac.uk.
}

Editorial group: Cochrane Metabolic and Endocrine Disorders Group.

Publication status and date: New, published in Issue 11, 2014.

Citation: McBain H, Mulligan K, Haddad M, Flood C, Jones J, Simpson A. Self-management interventions for type 2 diabetes in adult people with severe mental illness. Cochrane Database of Systematic Reviews 2014, Issue 11. Art. No.: CD011361. DOI: 10.1002/14651858.CD011361.

Copyright (C) 2014 The Cochrane Collaboration. Published by John Wiley \& Sons, Ltd.

\begin{abstract}
A B S T R A C T
This is the protocol for a review and there is no abstract. The objectives are as follows:

To assess the effects of diabetes self-management (DSM) interventions for people with type 2 diabetes with severe mental illness (SMI).
\end{abstract}

\section{B A C K G R O U N D}

\section{Description of the condition}

Diabetes is a common and serious global health problem, currently affecting an estimated $8.3 \%$ of adults - 382 million people worldwide, and taking up $11 \%$ of international health expenditure (International Diabetes Federation 2013). The condition typically develops in late adulthood, usually in people over the age of 40 years, but younger onset is becoming increasingly more common. The disease is characterised by poorly regulated blood glucose levels, which may arise from defects in insulin secretion (insulin deficiency), or in its action (insulin resistance), or both. Therefore the aim of treatment is to manage blood glucose levels to alleviate short-term symptoms and prevent or delay the development of long-term complications. Raised glucose in the blood, known as hyperglycaemia can initially be controlled by lifestyle management such as changes to diet and exercise, but given the progressive nature of type 2 diabetes it is likely that most individ- uals will ultimately require pharmacological intervention as well. This may initially be with oral hypoglycaemic drugs to increase the production or uptake of insulin and, if the disease remains uncontrolled, insulin therapy.

The primary symptoms of type 2 diabetes are increased thirst and urination, however, not all individuals will experience such symptoms and therefore many people remain undiagnosed for a sustained period of time. Undetected hyperglycaemia can have implications for the outcome of diabetes including greater risk of developing both macro- and microvascular complications. The primary microvascular complications to affect people with type 2 diabetes are in relation to the eyes, kidneys and nervous system, as well as coronary heart disease and major stroke (The Emerging Risk Factors Collaboration 2010).

Type 2 diabetes prevalence is increasing rapidly worldwide and is predicted to more than double in the years between 2000 and 2030 (Wild 2004). Although no single causal factor has been attributed to the development of the condition, increasing urbanization and ageing populations are strongly linked to global changes 
in diabetes incidence and prevalence. One important risk factor is a diagnosis of severe mental illness (SMI) such as schizophrenia, bipolar disorder and other psychoses, with research suggesting an almost two-fold increased risk of diabetes in people with SMI compared to those without SMI (Osborn 2008). This increased risk has been linked to a combination of factors including patient behaviour, in particular physical inactivity and poor diet, obesity (De Hert 2011) and higher rates of smoking (Lawrence 2009). Alongside lifestyle and behavioural factors, medications commonly prescribed for SMI are strongly associated with the development of metabolic abnormalities and weight gain which significantly increase the risk of type 2 diabetes (De Hert 2011). The World Health Organization (WHO) recognises mental disorder as being an important contributing factor to the global burden of non-communicable diseases such as diabetes and emphasises that equitable access to effective programmes and healthcare interventions is needed (WHO 2013a). As such, the WHO comprehensive mental health action plan for 2013 to 2020 states that developing good-quality mental health services requires the use of evidence-based protocols and practices. The plan suggests that health workers must not limit interventions to improving mental health but also attend to the physical health needs of people with a mental disorder and vice versa (WHO 2013b). In the UK, the Schizophrenia Commission (The Schizophrenia Commission 2012) and a report from the Royal College of Psychiatrists (Royal College of Psychiatrists 2009) also recognised that the poorer physical health of people with SMI needs to be urgently addressed and include amongst their advice the need for tailored health promotion programmes to help people to manage better their physical health, including any chronic illnesses.

Considering the importance of lifestyle in the management of type 2 diabetes, it is essential that patients possess the necessary skills to manage their condition. NICE guidelines for type 2 diabetes (NICE 2008) recommend that structured education is integrated into routine care and should be offered to all patients. In addition, the NHS report for the commissioning of mental health and diabetes services in the UK (NHS Diabetes 2011) clearly states that people with SMI who develop diabetes should have access to appropriate diabetes care. However, despite evidence to suggest that diabetes self-management (DSM) programs have a positive impact on clinical, lifestyle and psychosocial outcomes (Deakin 2005; Duke 2009; Pal 2013; Steed 2003; Steinsbekk 2012; Thorpe 2013), an examination of recent systematic reviews suggests that many trials either exclude those with SMI or do not specify the inclusion criteria in relation to SMI. In those that do not exclude on the basis of mental illness, there is no examination of whether a diagnosis of SMI has an impact on the effectiveness of such interventions.

For people with a SMI, physical health may be a low priority (Buhagiar 2011) and motivation to change may be limited, presenting additional challenges for successful self-management. It cannot therefore be assumed that the findings reported in existing systematic reviews of DSM interventions will generalise to those with SMI. A systematic review of DSM for those with schizophrenia and schizoaffective disorder found that approaches delivered in both inpatient and outpatient settings can be effective in managing type 2 diabetes, particularly those that address diet and exercise behaviour, but concluded that intervention packages need to be tailored to the unique challenges associated with decreased cognition and motivation, limited resources, as well as the loss of energy and weight gain associated with the use of antipsychotics (Cimo 2012).

\section{Description of the intervention}

DSM interventions are complex interventions as they consist of several interacting components (Craig 2008). Self-management refers to an individual's ability to manage the clinical and psychosocial consequences, along with the lifestyle changes inherent in living with a chronic condition (Barlow 2002). Due to this broad definition, the content and complexity of DSM interventions varies quite significantly, not only in terms of their aims and the behaviour/s they target (e.g. self-monitoring of blood glucose, insulin titration, diet or exercise), but also in terms of their intensity, duration, where the intervention is delivered (i.e. primary or secondary care), mode of delivery (i.e. group, individual, online), type and training of the facilitator (i.e. healthcare professional/s or lay person), the behaviour change techniques used and their theoretical background. Lorig 2003 has however defined five core self-management skills: (1) problem solving, (2) decision making, (3) use of healthcare resources, (4) forming a patient/healthcare professional partnership and (5) taking action.

\section{Adverse effects of the intervention}

There is little evidence to suggest that diabetes self-management interventions are associated with any adverse effects. However, it is possible that adverse effects could occur if:

- the content of the DSM intervention is not evidence-based possibly resulting in patients receiving incorrect information and training;

- patients misunderstand the information they are given or are unable to perform the required behaviours;

- patients became anxious as a result of being more engaged, for example if blood glucose readings are high and patients are unable to understand why (Peel 2004);

- being more engaged leads to inappropriate use of healthcare services;

- exercise leads to injury or increased pain and fatigue;

- patients make decisions which are detrimental to their health and well-being, such as insulin titration that leads to hypoglycaemia. 


\section{How the intervention might work}

The development of self-management interventions has been influenced by several theories of health behaviour change, including social cognitive theory (Bandura 1986), the theory of reasoned action and planned behaviour (Ajzen 1991), self-regulation theory (Leventhal 1984) and transtheoretical model (Prochaska 1997). These theories all identify concepts that predict health behaviour, primarily focusing on beliefs, attitudes and expectations. The resulting self-management interventions therefore differ in their theoretical underpinnings and hence the techniques they adopt to change behaviour. For example, a diabetes self-management intervention based on social cognitive theory (Bandura 1986) may seek to reduce carbohydrate intake by increasing diet-related selfefficacy. Bandura proposed a number of ways in which self-efficacy can be enhanced, including skills mastery where a person gains confidence by successfully achieving a goal, observation of someone performing the behaviour and verbal persuasion. These behaviour change techniques (BCTs) are the 'active ingredients' which explain how a self-management intervention works.

In response to guidance from the Medical Research Council (Craig 2008), which acknowledges the need for improved methods of specifying and reporting complex interventions, the Behaviour Change Technique Taxonomy (BCTTv1) (Michie 2013) was developed. The taxonomy provides standardised descriptions of the different techniques so that a shared language is used in the field of behaviour change, and links these techniques to published theories of behaviour change. The implementation of the BCTTv1 (Michie 2013) within this systematic review will therefore identify these active ingredients and provide a cumulative understanding, across the field of behaviour change, of how diabetes self-management interventions work.

In addition to the active ingredients there are a number of other key features of a behaviour change intervention including the behaviour or behaviours they aim to target (i.e. diet, exercise, selfmonitoring), duration, intensity, setting, mode of delivery and type and training facilitator, all of which can influence the efficacy and replicability of an intervention (Hoffman 2014). Figure 1 presents a simplified schematic representation of the conceptual framework for DSM which acknowledges the complex nature of these interventions, along with the most well-established selfmanagement behaviour change techniques included in these interventions. 
Figure I. Schematic representation of diabetes self-management

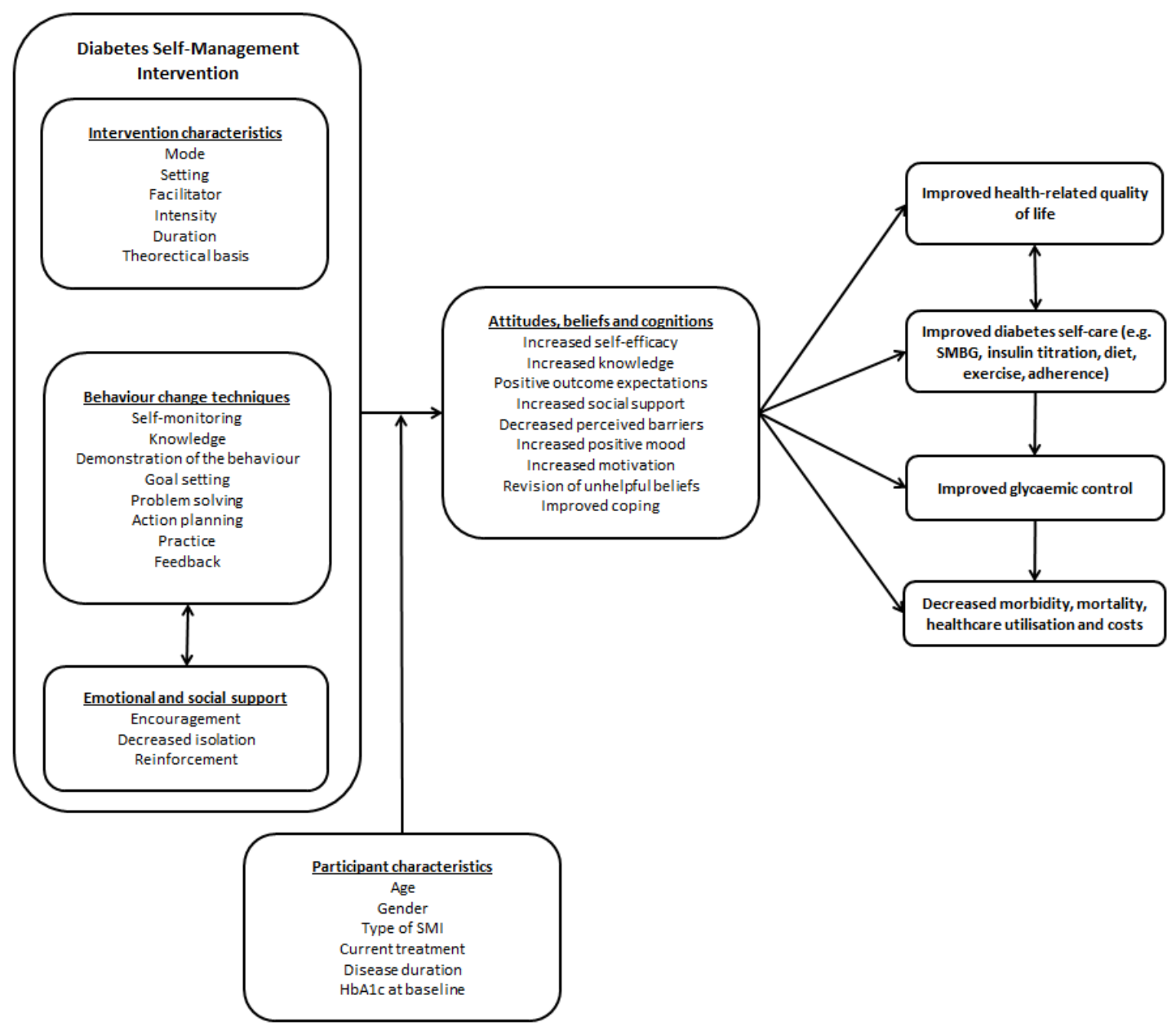

\section{Why it is important to do this review}

Whilst there is some evidence to indicate statistically and clinically significant benefits for DSM interventions in the general population (Deakin 2005; Duke 2009; Pal 2013; Steed 2003; Steinsbekk 2012; Thorpe 2013), there is little evidence that these interventions are effective in changing outcomes for people with SMI and type 2 diabetes. This review aims to broaden the inclusion criteria of a previous systematic review (Cimo 2012) to SMIs other than schizophrenia and schizoaffective disorder and other outcomes including patient-reported and socioeconomic outcomes.

This review will not only evaluate the effects of diabetes self-management interventions for people with SMI and type 2 diabetes, but will also provide us with the opportunity to describe, using established reporting systems, the active components of these interventions and the theoretical frameworks within which they were developed. The Medical Research Council (MRC) guidelines for developing complex interventions (Craig 2008) and the Consolidated Standards of Reporting Trials (CONSORT) statement for randomised controlled trials (RCTs) of non-pharmacological interventions (Boutron 2008) both acknowledge the need for improved methods of specifying and reporting intervention content. In addition to the BCTTv1 (Michie 2013), a coding system to assess the different ways that behavioural interventions have employed theory (Michie 2010) will also be applied. This theoretical coding system will enable an assessment of how, and to what extent, theory has been used to develop the intervention. Use of these coding systems will therefore be helpful in systematically identifying and documenting the content of DSM interventions for people with SMI and type 2 diabetes as well as establish which components and theories are most effective. By undertaking subgroup analysis this review will aim to identify not only if interven- 
tion effects vary by intervention characteristics, but also by patient characteristics, in order to establish what kind of self-management intervention works best for whom and under what conditions.

\section{O B J E C T I V E S}

To assess the effects of diabetes self-management (DSM) interventions for people with type 2 diabetes with severe mental illness (SMI).

\section{METHODS}

\section{Criteria for considering studies for this review}

\section{Types of studies}

We will include RCTs.

\section{Types of participants}

Adult individuals with SMI and type 2 diabetes. Adults participants will be defined as those aged 18 years and over.

\section{Diagnostic criteria}

Diagnosis of type 2 diabetes should have been consistent with the standard classification criteria valid at the time of the trial (for example ADA 1999; ADA 2008; WHO 1998). We plan to subject type 2 diabetes mellitus diagnostic criteria to a sensitivity analysis. SMI will be defined as psychosis, schizophrenia, schizoaffective disorder, bipolar disorder, personality disorder, or depression with psychotic features, however diagnosed.

\section{Types of interventions}

\section{Intervention}

Interventions that are targeted to improve the self-management of type 2 diabetes mellitus; this could include interventions that target self-monitoring of blood glucose, diet or exercise behaviour. This may or may not include the self-management of SMI but interventions which focus solely on the management of SMI will be excluded. The intervention can be of any duration.

\section{Comparator}

The comparison group will be either another active intervention or usual/standard care.

\section{Exclusions}

Any intervention which:

- includes only participants with type 1 diabetes;

- includes participants without SMI;

- involves participants under the age of 18 years, including trials that include both adults and children;

- are targeted at healthcare professionals;

- focuses exclusively on self-management of SMI.

Studies that include both type 1 and 2 diabetes will only be included if results can be extracted for participants with type 2 diabetes.

\section{Types of outcome measures}

\section{Primary outcomes}

- Self-care behaviours.

- Diabetes-related complications.

- Adverse events.

\section{Secondary outcomes}

- All-cause mortality.

- Health-related quality of life.

- Diabetes knowledge.

- Self-efficacy.

- Progression of severe mental illness.

- HbAlc.

- Body mass index (BMI).

- Weight.

- Blood pressure.

- Change in medication or intensity of drug treatment.

- Socioeconomic effects.

\section{Method of outcome measurement}

- Diabetes-related complications defined as vascular complications (angina pectoris, myocardial infarction, stroke or peripheral vascular disease), neuropathy, nephropathy, retinopathy, diabetic foot and lower limb amputation and heart failure.

- Glycaemic control will be measured via HbA1c.

- Adverse events of the intervention defined as hypoglycaemia, pain, fatigue and anxiety.

- All-cause mortality defined as death from any cause.

- Health-related quality of life evaluated with a validated generic or disease specific instrument such as the SF-36® (McHorney 1993; Ware 1992) or Diabetes Health Profile (DHP) (Meadows 2000). 
- Self-care behaviours evaluated with a validated instrument such as the Summary of Diabetes Self-care Activities (SDSCA) measure (Toobert 2000).

- Diabetes knowledge evaluated with a validated instrument such as the Brief Diabetes Knowledge Test (Fitzgerald 1998).

- General or diabetes-specific self-efficacy evaluated with a validated instrument such as the Diabetes Empowerment Scale (Anderson 2000).

- Progression of severe mental illness assessed using a diseasespecific such as the Positive and Negative Syndrome Scale (Kay 1987) or generic such as the Clinical Global Impressions scale (Busner 2007) or Health of the Nation Outcome Scale (Wing 1998).

- BMI in $\mathrm{kg} / \mathrm{m}^{2}$ or waist-to-hip ratio as a ratio.

- Weight in $\mathrm{kg}$ or lbs.

- Systolic and diastolic blood pressure in $\mathrm{mm} \mathrm{Hg}$.

- Intensity of type 2 diabetes treatment defined as an increase in medication dose or the introduction of an additional drug.

- Intensity of SMI treatment defined as an increase in medication dose or the introduction of an additional drug.

- Socioeconomic effects: direct costs defined as admission/ readmission rates, average length of stay, visits to general practitioner, accident/emergency visits; indirect costs: defined as resources lost due to illness by patient or their family member.

\section{Timing of outcome measurement}

The timing of outcome measurement will be classified as short, medium and long term. For any meta-analysis performed, data from the longest follow-up will be used. Short-term follow-up will be defined as measurement taken within one month of the end of the intervention period and will therefore capture the immediate effects of the intervention; medium-term follow-up will be defined as between one and six months post intervention and long-term follow-up six months and longer.

\section{'Summary of findings' table}

We will present a 'Summary of findings' table reporting the following outcomes.

1. Diabetes-related complications.

2. All-cause mortality.

3. Adverse events.

4. Health-related quality of life.

5. Self-care behaviours.

6. HbA1c.

7. Socioeconomic effects.

\section{Search methods for identification of studies}

\section{Electronic searches}

We will search the following sources from inception of each database to the specified date and will place no restrictions on the language of publication.

- The Cochrane Library.

- CDSR

- CENTRAL

- DARE

- HTA

- MEDLINE.

- EMBASE.

- PsycINFO.

- Allied and Complimentary Medicine Database (AMED).

- Health Technology Assessment (HTA).

- NHS Economic Evaluations Database (NHS EED).

- CINAHL Plus.

- ICTRP trial register (http://apps.who.int/trialsearch/)

- Australian New Zealand Clinical Trials Registry

- ClinicalTrials.gov

- EU Clinical Trials Register

- ISRCTN

- Brazilian Clinical Trials Registry

- Chinese Clinical Trial Registry

- Clinical Trials Registry - India

- Clinical Research Information Service - Republic of

Korea

- Cuban Public Registry of Clinical Trials

- German Clinical Trials Register

- Iranian Registry of Clinical Trials

- Japan Primary Registries Network

- Pan African Clinical Trial Registry

- Sri Lanka Clinical Trials Registry

- The Netherlands National Trial Register

- Thai Clinical Trials Register

We will continuously apply a MEDLINE (via Ovid SP) email alert service to identify newly published studies using the same search strategy as described for MEDLINE (for details on search strategies see Appendix 1). After supplying the final review draft for editorial approval, the Cochrane Metabolic and Endocrine Disorders Group (CMED) will perform a complete update search on all databases available at the editorial office and send the results to the review authors. Should we identify new studies for inclusion we will evaluate these, incorporate findings in our review and resubmit another review draft (Beller 2013).

If we detect additional relevant key words during any of the electronic or other searches, we will modify the electronic search strategies to incorporate these terms and document the changes.

\section{Searching other resources}

We will try to identify other potentially eligible trials or ancillary publications by searching the reference lists of retrieved articles including trials, (systematic) reviews, meta-analyses and health tech- 
nology assessment reports. Unpublished literature will be searched for using the following databases.

- BASE: Bielefeld Academic Research Engine (http:// www.base-search.net/).

- Open Grey (http://www.opengrey.eu/).

- NHS Evidence (http://www.evidence.nhs.uk/).

- UK Clinical Research Network Study Portfolio (http:// public.ukcrn.org.uk/search/).

\section{Data collection and analysis}

\section{Selection of studies}

Two review authors (HM, MH) will independently scan the abstract, title, or both, of every record retrieved, to determine which studies should be assessed further. We will investigate all potentially-relevant articles as full text. We will resolve any discrepancies through consensus or recourse to a third review author (CF). If resolving disagreement is not possible, the article will be added to those 'awaiting assessment' and we will contact study authors for clarification. We will present an adapted PRISMA (Preferred Reporting Items for Systematic Reviews and Meta-Analyses) diagram of study selection (Liberati 2009).

\section{Data extraction and management}

For studies that fulfil inclusion criteria, two review authors (HM, $\mathrm{KM}$ ) will independently extract key participant and intervention characteristics and report data on efficacy outcomes and adverse events using standard data extraction templates as supplied by the CMED, with any disagreements to be resolved by discussion, or, if required, by consultation with a third review author(AS).

We will provide information including trial identifier about potentially relevant ongoing studies in the table 'Characteristics of ongoing studies'. We will try to find the protocol of each included study and will report primary, secondary and other outcomes in comparison with data in publications in a joint appendix 'Matrix of study endpoint (publications and trial documents)'.

We will email all authors of included studies to enquire whether they are willing to answer questions regarding their trials. We will present the results of this survey in an appendix. Thereafter, we will seek relevant missing information on the trial from the primary author(s) of the article, if required.

Both the intervention and comparator group will be coded for the use of theory and behaviour change techniques. This will enable articles to be grouped according to the unique components found within the diabetes self-management (DSM) intervention:

\section{Use of theory}

A theory coding scheme has been developed which assesses how and to what extent theory has been used to develop an intervention (Michie 2010). The coding scheme consists of 19 items each requiring a yes, no or do not know response. These 19 questions are classified into six categories: (i) is theory mentioned, (ii) are the relevant theoretical constructs targeted, (iii) is theory used to select recipients or tailor an intervention, (iv) are the relevant theoretical constructs measured, (v) is theory tested and (vi) has theory been refined. For the purposes of any statistical analysis, if the theoretical basis for the intervention group is the same as the control group, the intervention will be coded as not having a theoretical basis (except for descriptive purposes); as theory would be unable to explain the difference in the effect size between the two groups.

\section{Behaviour change techniques}

The recently published behaviour change technique taxonomy (BCTTv1) (Michie 2013) will be used to code both the intervention and control groups. The behaviour change technique (BCT) will either be coded as being present within the intervention and control group, in the intervention group only or within the control group only. A description will be provided of the most common and consistently implemented techniques. For the purposes of any statistical analysis only those BCTs which are unique to the intervention groups will be analysed to enable identification of those techniques that could be attributed to differences in outcomes between groups. Those involved in the extraction and coding of data on BCTs will have undertaken the required BCTT training. If the same BCT is employed within both the intervention and control group, the intervention will be coded as not containing the BCT (except for descriptive purposes); as the BCT would be unable to explain any differences in effect size between the two conditions.

\section{Dealing with duplicate and companion publications}

In the event of duplicate publications, companion documents or multiple reports of a primary study, we will maximise yield of information by collating all available data and use the most complete data set aggregated across all known publications. In case of doubt, the publication reporting the longest follow-up associated with our primary or secondary outcomes will be given priority.

\section{Assessment of risk of bias in included studies}

Two review authors (HM, JJ) will assess the risk of bias of each included study independently. We will resolve disagreements by consensus, or by consultation with a third author $(\mathrm{MH})$. We will assess risk of bias using the Cochrane Collaboration's tool for assessment of risk of bias (Higgins 2011a; Higgins 2011b) using the following criteria.

- Random sequence generation (selection bias). 
- Allocation concealment (selection bias).

- Blinding (performance bias and detection bias), blinding of participants and personnel assessed separately from blinding of outcome assessment.

- Incomplete outcome data (attrition bias).

- Selective reporting (reporting bias).

- Other bias.

We will judge 'Risk of bias' criteria as 'low risk', 'high risk' or 'unclear risk' and will evaluate individual bias items as described in the Cochrane Handbook for Systematic Reviews of Interventions (Higgins 2011a). We will present a 'Risk of bias' graph and a 'Risk of bias summary' figure. We will assess the impact of individual bias domains on study results at the endpoint and study levels. In case of high risk of selection bias, all endpoints investigated in the associated study will be marked as 'high risk'.

For performance bias (blinding of participants and personnel) and detection bias (blinding of outcome assessors) we will evaluate the risk of bias separately for each outcome (Hróbjartsson 2013). We will note whether outcomes were measured subjectively or objectively, for example, if body weight was measured by participants or study personnel.

We will consider the implications of missing outcome data from individual participants per outcome such as high drop-out rates (e.g. above $15 \%$ ) or disparate attrition rates (e.g. difference of $10 \%$ or more between study arms).

We will assess outcome reporting bias by integrating the results of an appendix 'Examination of outcome reporting bias' (Kirkham 2010), an appendix 'Matrix of study endpoints (publications and trial documents)' and section 'Outcomes (outcomes reported in abstract of publication)' of the 'Characteristics of included studies' table. This analysis will form the basis of the judgement of selective reporting (reporting bias).

We define the following endpoints as subjective outcomes.

- Health-related quality of life.

- Self-care behaviours.

- Diabetes knowledge.

- Self-efficacy.

- Adverse events, depending on measurement.

- Body mass index (BMI), depending on measurement.

- Weight, depending on measurement.

- Change in medication or intensity of drug treatment, depending on measurement.

We define the following outcomes as objective outcomes.

- HbAlc.

- All-cause mortality.

- Diabetes-related complications.

- Body mass index (BMI), depending on measurement.

- Weight, depending on measurement.

- Blood pressure.

- Change in medication or intensity of drug treatment, depending on measurement.
- Socioeconomic effects.

\section{Measures of treatment effect}

We will express dichotomous outcomes as risk ratios, with $95 \%$ confidence intervals (CI). For continuous outcomes where the same measurement scale has been used e.g. HbA1c, the measure of treatment effect will be the mean difference or difference in means, with $95 \%$ CI. For continuous outcomes where different measurement scales have been used, such as quality of life, the measure of treatment effect will be standardised mean difference (SMD). The definition of SMD used in Cochrane reviews is the effect size known in social science as Hedges' g (adjusted) (Hedges 1985). If Hedge's $g$ is not reported it will be calculated as the difference between the two means (intervention and control) divided by the pooled standard deviation. If this is not possible, the results of each study will be described in a narrative synthesis. We will express time-to-event data as hazard ratios (HRs) with 95\% CIs.

\section{Unit of analysis issues}

We will take into account the level at which randomisation occurred, such as cross-over trials, cluster-randomised trials and multiple observations for the same outcome.

Data from cross-over trials will be extracted for the intervention and control group at baseline and at the time point immediately prior to cross-over. In case of a unit of analysis error occurrence in cluster-randomised controlled trials, we will adjust for the design effect by reducing the size of the trial to its "effective sample size" (Rao 1992). This is calculated by dividing the original sample size by the 'design effect'. The design effect is $1+(\mathrm{M}-1) *$ ICC, where $\mathrm{M}$ is the average cluster size and ICC is the intra-cluster correlation coefficient. For dichotomous data, both the number of participants and the number experiencing the event will be divided by the design effect. For continuous data, only the sample sizes will be reduced; means and standard deviations will remain unchanged (Higgins 2011a).

\section{Dealing with missing data}

We will obtain missing data from authors, if feasible, and carefully evaluate important numerical data such as screened, randomised participants as well as intention-to-treat (ITT), and as-treated and per-protocol populations. We will investigate attrition rates, e.g. drop-outs, losses to follow-up and withdrawals, and critically appraise issues of missing data or using imputation methods (e.g. last observation carried forward (LOCF), mean imputation, imputing based on predicted values from a regression analysis).

Where standard deviations for outcomes are not reported and we do not receive information from study authors, we will impute these values by assuming the standard deviation of the missing outcome to be the average of the standard deviations from those studies where this information was reported. We will investigate 
the impact of imputation on meta-analyses by means of sensitivity analysis.

If authors fail to respond within one month of first contact, a second attempt will be made. If after two months no response is received data will be recorded as missing.

\section{Assessment of heterogeneity}

In the event of substantial clinical, methodological or statistical heterogeneity, we will not report study results as the pooled effect estimate in a meta-analysis. We will identify heterogeneity (inconsistency) by visual inspection of the forest plots and by using a standard $\mathrm{Chi}^{2}$ test with a significance level of $\alpha=0.1$. In view of the low power of this test, we will also consider the $\mathrm{I}^{2}$ statistic, which quantifies inconsistency across studies to assess the impact of heterogeneity on the meta-analysis (Higgins 2002; Higgins 2003); where an $I^{2}$ statistic of $75 \%$ or more indicates a considerable level of heterogeneity (Higgins 2011a). We expect type of diabetes treatment (i.e. insulin versus non-insulin dependent type 2 diabetes) and SMI diagnosis to introduce clinical heterogeneity.

\section{Assessment of reporting biases}

If we include 10 studies or more that investigate a particular outcome, we will use funnel plots to assess small-study effects. Owing to several possible explanations for funnel plot asymmetry, we will interpret results carefully (Sterne 2011).

\section{Data synthesis}

Unless there is good evidence for homogeneous effects across studies, we will summarise primarily low risk of bias data by means of a random-effects model (Wood 2008). We will interpret randomeffects meta-analyses with due consideration of the whole distribution of effects, ideally by presenting a prediction interval (Higgins 2009). A prediction interval specifies a predicted range for the true treatment effect in an individual study and will be calculated if there are at least three studies (Riley 2011). In addition, we will perform statistical analyses according to the statistical guidelines contained in the latest version of the Cochrane Handbook for Systematic Reviews of Interventions (Higgins 2011a).

\section{Quality of evidence}

We will present the overall quality of the evidence for each outcome according to the Grading of Recommendations Assessment, Development and Evaluation (GRADE) approach, which takes into account issues not only related to internal validity (risk of bias, inconsistency, imprecision, publication bias), but also to external validity such as directness of results. Two review authors (HM, $\mathrm{KM}$ ) will independently rate the quality for each outcome. We will present a summary of the evidence in a 'Summary of findings' table, which provides key information about the best estimate of the magnitude of the effect, in relative terms and absolute differences for each relevant comparison of alternative management strategies, numbers of participants and studies addressing each important outcome and the rating of the overall confidence in effect estimates for each outcome. We will create the 'Summary of findings' table based on the methods described in chapter 11 of the Cochrane Handbook for Systematic Reviews of Interventions (Higgins 2011a). We will present results on the outcomes as described in the Types of outcome measures section. If meta-analysis is not possible, we will present results in a narrative 'Summary of findings' table. In addition, we will establish an appendix 'Checklist to aid consistency and reproducibility of GRADE assessments' (Meader 2014) to help with standardisation of 'Summary of findings' tables.

\section{Subgroup analysis and investigation of heterogeneity}

Patient education and self-management are an integral part of diabetes care. Patients have a right to receive education about their diabetes and the treatment options, as well as information and training in how they can best manage their condition. Clearly the efficacy of diabetes self-management interventions for people with SMI is important, but it is equally important to identify the optimal content and delivery methods as well as the participant characteristics which lead to the most improved outcomes. Subgroup analyses will therefore be performed in order to establish if intervention effects vary with different participant populations or intervention characteristics. These comparisons will be hypothesis generating only.

- Age.

- Gender.

- Disease duration at baseline for both type 2 diabetes and SMI.

- Insulin-treated versus non-insulin treated type 2 diabetes.

- SMI treatment (i.e. antipsychotic medication versus no antipsychotic medication, typical (first generation) antipsychotic medication versus atypical (second generation) antipsychotic medication, olanzapine or clozapine treatment versus other antipsychotic treatment).

- SMI diagnosis (i.e. psychosis, schizophrenia, schizoaffective disorder, bipolar disorder, personality disorder or depression with psychotic features).

- Targeted behaviour (e.g. self-monitoring, self-adaption of drug/insulin, exercise, diet).

- HbA1c at baseline.

- Behaviour change techniques used.

- Use of a theory to inform the intervention.

- Intensity of the intervention.

- Intervention setting (i.e. primary, secondary care or community).

\section{Sensitivity analysis}


We will perform sensitivity analyses in order to explore the influence of the following factors (when applicable) on effect sizes.

- Restricting the analysis to published studies.

- Restricting the analysis by taking into account risk of bias, as specified in the section 'Assessment of risk of bias in included studies'.

- Restricting the analysis to very long or large studies to establish the extent to which they dominate the results.

- Restricting the analysis to studies using the following filters: diagnostic criteria, imputation, language of publication, source of funding (industry versus other), country.
We will also test the robustness of the results by repeating the analysis using different measures of effect size (RR, OR etc.) and different statistical models (fixed-effect and random-effects models).

\section{ACKNOWLEDGEMENTS}

We thank Karla Bergerhoff and Inti-Maria Metzendorf the former and current Trials Search Coordinator in the Cochrane Metabolic and Endocrine Disorders Group for assistance in designing the search strategy.

\section{R E F E R E N C E S}

\section{Additional references}

\section{ADA 1999}

The Expert Committee on the Diagnosis and Classification of Diabetes Mellitus. Report of the expert committee on the diagnosis and classification of diabetes mellitus. Diabetes Care 1999;22:S5-19.

\section{ADA 2008}

American Diabetes Association. Standards of medical care in diabetes - 2008. Diabetes Care 2008;31:S12-54.

\section{Ajzen 1991}

Ajzen I. The theory of planned behavior. Organisational Behavior and Human Decision Processes 1991;50:179-211.

Anderson 2000

Anderson RM, Funnell MM, Fitzgerald JT, Marrero DG. The Diabetes Empowerment Scale: a measure of psychosocial self-efficacy. Diabetes Care 2000;23:739-43.

Bandura 1986

Bandura A. Social. Social Foundations of Thought and Action: a Social Cognitive Theory. Prentice-Hall, 1986.

\section{Barlow 2002}

Barlow JH. How to use education as an intervention in osteoarthritis. Balliere's Best Practice \& Research. Clinical Rheumatology 2001;15(4):545-8.

\section{Beller 2013}

Beller EM, Chen JK, Wang UL, Glasziou PP. Are systematic reviews up-to-date at the time of publication?. Systematic Reviews 2013;2:36.

\section{Boutron 2008}

Boutron I, Moher D, Altman DG, Schulz K, Ravaud P for the CONSORT group. Extending the CONSORT statement to randomized trials of nonpharmacologic treatment: explanation and elaboration. Annals of Internal Medicine 2008;148:295-309.

\section{Buhagiar 2011}

Buhagiar K, Parsonage L, Osborn DP. Physical health behaviours and health locus of control in people with schizophrenia-spectrum disorder and bipolar disorder: a cross-sectional comparative study with people with nonpsychotic mental illness. BMC Psychiatry 2011;11:104.

\section{Busner 2007}

Busner J, Targum SD. The clinical global impressions scale: applying a research tool in clinical practice. Psychiatry 2007; 4(7):28-37.

\section{Cimo 2012}

Cimo A, Stergiopoulos E, Cheung C, Bonato S, Dewa CS. Effective lifestyle interventions to improve type II diabetes self-management for those with schizophrenia or schizoaffective disorder. BMC Psychiatry 2012;12:24.

\section{Craig 2008}

Craig P, Dieppe P, Macintrye S, Michie S, Nazareth I, Petticrew M. Developing and evaluating complex interventions: the new Medical Research Council guidance. BMJ 2008;337:a1655.

\section{De Hert 2011}

De Hert M, Correll CU, Bobes J, Cetkovich-Bakmas M, Cohen D, Asai I, et al.Physical illness in patients with severe mental disorders. I. Prevalence, impact of medications and disparities in health care. World Psychiatry 2011;10(1): 52-77.

\section{Deakin 2005}

Deakin TA, McShane CE, Cade JE, Williams R. Group based training for self-management strategies in people with type 2 diabetes mellitus. Cochrane Database of Systematic Reviews 2005, Issue 2. [DOI: 10.1002/ 14651858.CD003417.pub2]

\section{Duke 2009}

Duke SAS, Colagiuri S, Colagiuri R. Individual patient education for people with type 2 diabetes mellitus. Cochrane Database of Systematic Reviews 2009, Issue 1. [DOI: 10.1002/14651858.CD005268.pub2]

\section{Fitzgerald 1998}

Fitzgerald JT, Funnell MM, Hess GE, Barr PA, Anderson RM, Hiss RG, et al.The reliability and validity of a brief diabetes knowledge test.. Diabetes Care 1998;21:706-10. 


\section{Hedges 1985}

Hedges LV, Olkin I. Statistical Methods for Meta-analysis. New York: Academic Press, 1985.

Higgins 2002

Higgins JPT, Thompson SG. Quantifying heterogeneity in a meta-analysis. Statistics in Medicine 2002;21:1539-58.

Higgins 2003

Higgins JPT, Thompson SG, Deeks JJ, Altman DG. Measuring inconsistency in meta-analysis. BMJ 2003;327: 557-60.

Higgins 2009

Higgins JPT, Thompson SG, Spiegelhalter DJ. A reevaluation of random-effects meta-analysis. Journal of the Royal Statistical Society: Series A (Statistics in Society) 2009; 172:137-59.

Higgins 2011a

Higgins JPT, Green S (editors). Cochrane Handbook for Systematic Reviews of Interventions Version 5.1.0 [updated March 2011]. The Cochrane Collaboration, 2011. Available from www.cochrane-handbook.org.

\section{Higgins 2011b}

Higgins JPT, Altman DG, Gøtzsche PC, Jüni P, Moher D, Oxman AD, et al.The Cochrane Collaboration's tool for assessing risk of bias in randomised trials. BMJ 2011;343: d5928.

Hoffman 2014

Hoffman TC, Glasziou PP, Boutron I, Milne R, Perera R, Moher $\mathrm{D}$, et al.Better reporting of interventions: template for intervention description and replication (TIDieR) checklist and guide. BMJ 2014;348:g1687.

\section{Hróbjartsson 2013}

Hróbjartsson A, Thomsen AS, Emanuelsson F, Tendal B, Hilden J, Boutron I, et al.Observer bias in randomized clinical trials with measurement scale outcomes: a systematic review of trials with both blinded and nonblinded assessors. Canadian Medical Association Journal 2013;185:E201-11.

International Diabetes Federation 2013

International Diabetes Federation. IDF Diabetes Atlas $6^{\text {th }}$ edition. http://www.idf.org/diabetesatlas. Accessed 2 May 2014 .

Kay 1987

Kay SR, Flszbein A, Opfer LA. The Positive and Negative Syndrome Scale (PANSS) for Schizophrenia. Schizophrenia Bulletin 1987;13(2):261-76.

\section{Kirkham 2010}

Kirkham JJ, Dwan KM, Altman DG, Gamble C, Dodd $\mathrm{S}$, Smyth R, et al.The impact of outcome reporting bias in randomised controlled trials on a cohort of systematic reviews. BMJ 2010;340:c365.

\section{Lawrence 2009}

Lawrence D, Mitrou F, Zubrick SR. Smoking and mental illness: results from population surveys in Australia and the United States. BMC Public Health 2009;9:285.

\section{Leventhal 1984}

Leventhal H, Nerenz DR, Steele DF. Illness representations and coping with health threats. A Handbook of Psychology and Health. Baum A, Singer J. Hillsdales, NJ: Erlbaum, 1984:219-52.

\section{Liberati 2009}

Liberati A, Altman DG, Tetzlaff J, Mulrow C, Gøtzsche PC, Ioannidis JPA, et al.The PRISMA statement for reporting systematic and meta-analyses of studies that evaluate interventions: explanation and elaboration. PLoS Medicine 2009;6:1-28.

Lorig 2003

Lorig KR, Holman H. Self-management education: history, definition, outcomes, and mechanisms. Annals of Behavioural Medicine 2003;26:1-7.

\section{McHorney 1993}

McHorney CA, Ware JE, Raczek AE. The MOS 36-item short-form health survey: II. Psychometric and clinical tests of validity in measuring physical and mental health constructs. Medical Care 1993;31:247-63.

\section{Meader 2014}

Meader N, King K, Llewellyn A, Norman G, Brown J, Rodgers $\mathrm{M}$, et al.A checklist designed to aid consistency and reproducibility of GRADE assessments: development and pilot validation. Systematic Reviews 2014;3:82.

\section{Meadows 2000}

Meadows KA, Abrams C, Sandbaek A. Adaptation of the Diabetes HealthProfile (DHP-1) for use with patients with Type 2 Diabetes mellitus: psychometric evaluation and cross-cultural comparison. Diabetic Medicine 2000;17: $572-80$.

\section{Michie 2010}

Michie S, Prestwich A. Are interventions theory based? Development of a theory coding scheme. Health Psychology 2010;29:1-8.

\section{Michie 2013}

Michie S, Johnston M, Abraham C, Francis J, Eccles MP. The behaviour change technique taxonomy (v1) of 93 hierarchically clustered techniques: building an international consensus for the reporting of behaviour change interventions. Annals of Behavioural Medicine 2013; 46:81-95.

\section{NHS Diabetes 2011}

NHS Diabetes. Commissioning Mental Health and Diabetes Services. London: NHS Diabetes, 2011.

\section{NICE 2008}

National Collaborating Centre for Chronic Conditions. Type 2 Diabetes: National Clinical Guideline for Management in Primary and Secondary Care (update). London: Royal College of Physicians, 2008.

\section{Osborn 2008}

Osborn D, Wright C, Levy G, King M, Deo R, Nazareth I. Relative risk of diabetes, dyslipidaemia, hypertension and the metabolic syndrome in people with severe mental illnesses: systematic review and meta analysis. $B M C$ Psychiatry 2008;8:84.

\section{Pal 2013}

Pal K, Eastwood SV, Michie S, Farmer AJ, Barnard ML, Peacock R, et al.Computer-based diabetes self-management 
interventions for adults with type 2 diabetes mellitus. Cochrane Database of Systematic Reviews 2013, Issue 3. [DOI: 10.1002/14651858.CD008776.pub2]

\section{Peel 2004}

Peel E, Parry O, Douglas M, Lawton J. Blood glucose self-monitoring in non-insulin-treated type 2 diabetes: a qualitative study of patients' perspectives. British Journal of General Practice 2004;54:183-8.

\section{Prochaska 1997}

Prochaska JO, Velicer WF. The transtheoretical model of health behaviour change. American Journal of Health Promotion 1997;12:38-48.

Rao 1992

Rao JNK, Scott AJ. A simple method for the analysis of clustered binary data. Biometrics 1992;48:577-85.

Riley 2011

Riley RD, Higgins JP, Deeks JJ. Interpretation of random effects meta-analyses. BMJ 2011;342:d549.

Royal College of Psychiatrists 2009 Royal College of Psychiatrists. OP67 Physical Health in Mental Health: Final Report of a Scoping Group. London: Royal College of Psychiatrists, 2009.

\section{Steed 2003}

Steed L, Cooke D, Newman S. A systematic review of psychosocial outcomes following education, selfmanagement and psychological interventions in diabetes mellitus. Patient Education and Counseling 2003;51:5-15.

\section{Steinsbekk 2012}

Steinsbekk A, Rygg L, Lisulo M, Rise MB, Fretheim A. Group based diabetes self-management education compared to routine treatment for people with type 2 diabetes mellitus. A systematic review and meta-analysis. BMC Health Services Research 2012;12:213.

Sterne 2011

Sterne JA, Sutton AJ, Ioannidis JP, Terrin N, Jones DR, Lau $\mathrm{J}$, et al.Recommendations for examining and interpreting funnel plot asymmetry in meta-analyses of randomised controlled trials. BMJ 2011;343:d4002.

The Emerging Risk Factors Collaboration 2010 The Emerging Risk Factors Collaboration. Diabetes mellitus, fasting blood glucose concentration, and risk of vascular disease: a collaborative meta-analysis of 102 prospective studies. Lancet 2010;375:2215-22.

\section{The Schizophrenia Commission 2012}

The Schizophrenia Commission. The Abandoned Illness: a Report by the Schizophrenia Commission. London: Rethink Mental Illness, 2012.

\section{Thorpe 2013}

Thorpe CT, Fahey LE, Johnson H, Deshpande M, Thorpe JM, Fisher EB. Facilitating healthy coping in patients with diabetes: a systematic review. Diabetes Educator 2013;39: $33-52$.

\section{Toobert 2000}

Toobert DJ, Hampson SE, Glasgow RE. The summary of diabetes self-care activities measure: results from 7 studies and a revised scale. Diabetes Care 2000;23:943-50.

\section{Ware 1992}

Ware JE, Sherbourne CD. The MOS 36-item short-form health survey(SF-36): I. Conceptual framework and item selection. Medical Care 1992;30(6):473-83.

\section{WHO 1998}

Alberti KM, Zimmet PZ. Definition, diagnosis and classification of diabetes mellitus and its complications. Part I: diagnosis and classification of diabetes mellitus. Provisional report of a WHO consultation. Diabetic Medicine 1998;15:539-53.

\section{WHO 2013a}

World Health Organization. Global action plan for the prevention and control of noncommunicable diseases 20132020. WHO Press.

\section{WHO 2013b}

World Health Organization. Mental Health Action Plan 2013-2020. WHO Press.

\section{Wild 2004}

Wild S, Roglie G, Green A, Sicree R, King, H. Global prevalence of diabetes estimates for the year 2000 and projections for 2030. Diabetes Care 2004;27:1047-53.

\section{Wing 1998}

Wing KJ, Beevor AS, Curtis RH, Park SB, Hadden S, Burns A. Health of the Nation Outcome Scale (HoNOS). Research and development. British Journal of Psychiatry 1998;172:11-8.

\section{Wood 2008}

Wood L, Egger M, Gluud LL, Schulz KF, Juni P, Altman DG, et al.Empirical evidence of bias in treatment effect estimates in controlled trials with different interventions and outcomes: meta-epidemiological study. BMJ 2008; 336:601-5.

* Indicates the major publication for the study 
A P PENDICES

\section{Appendix I. Search strategies}

\section{Search terms and databases}

Unless otherwise stated, search terms are free text terms.

Abbreviations:

'\$': stands for any character; '?': substitutes one or no character; adj: adjacent (i.e. number of words within range of search term); exp: exploded MeSH; MeSH: medical subject heading (MEDLINE medical index term); pt: publication type; sh: MeSH; tw: text word

\section{The Cochrane Library}

\# 1MeSH descriptor Diabetes mellitus, type 2 explode all trees

\# 2(MODY in All Text or NIDDM in All Text or TDM2 in All Text or TD2 in All Text)

\# 3((non in All Text and insulin* in All Text and depend* in All Text) or (noninsulin* in All Text and depend* in All Text) or (non in All Text and insulindepend* in All Text) or noninsulindepend* in All Text)

\# 4(typ? in All Text and (2 in All Text near/6 diabet* in All Text))

\# 5(typ? in All Text and (II in All Text near/6 diabet* in All Text))

\# 6(adult* in All Text near/6 diabet* in All Text)

\# 7 (matur* in All Text near/6 diabet* in All Text)

\# 8(late in All Text near/6 diabet* in All Text)

\# 9(slow in All Text near/6 diabet* in All Text)

\# 10(stabl* in All Text near/6 diabet* in All Text)

$\# 11$ (\#1 or $\# 2$ or $\# 3$ or $\# 4$ or $\# 5$ or $\# 6$ or $\# 7$ or $\# 8$ or $\# 9$ or $\# 10$ )

\# 12MeSH descriptor Diabetes insipidus explode all trees

\# 13(diabet* in All Text and insipidus in All Text)

\# 14(\#12 or \#13)

\# 15(\#11 and not \#14)

\# 16MeSH descriptor Mental disorders explode all trees

\# 17MeSH descriptor Psychotic disorders explode all trees

\# 18MeSH descriptor Mood disorders explode all trees

\# 19MeSH descriptor Personality disorders explode all trees

\# 20MeSH descriptor Bipolar disorder explode all trees

\# 21MeSH descriptor Schizophrenia explode all trees

\# 22((mental in All Text near/6 disorder* in All Text) or (mental in All Text near/6 illness in All Text))

\# 23(schizo* in All Text or psycho* in All Text)

\# 24((bipolar in All Text near/6 disorder* in All Text) or (affective in All Text near/6 disorder* in All Text) or (personality in All Text near/6 disorder* in All Text))

\# 25MeSH descriptor Depression explode all trees

\# 26depression* in All Text

$\# 27$ (\#16 or \#17 or \#18 or \#19 or \#20 or \#21 or \#22 or \#23 or \#24 or \#25 or \#26)

\# 28(\#15 and \#27)

\# 29MeSH descriptor Patient education as topic explode all trees

\# 30MeSH descriptor Patient compliance explode all trees

\# 31MeSH descriptor Self care explode all trees

\# 32MeSH descriptor Health promotion explode all trees

\# 33MeSH descriptor Behavior therapy explode all trees

\# 34MeSH descriptor Health behavior explode all trees

Self-management interventions for type 2 diabetes in adult people with severe mental illness (Protocol)

Copyright @ 2014 The Cochrane Collaboration. Published by John Wiley \& Sons, Ltd. 


\section{(Continued)}

\# 35MeSH descriptor Program evaluation explode all trees

\# 36MeSH descriptor Life style explode all trees

\# 37((self in All Text near/6 care in All Text) or (self in All Text near/6 monitoring in All Text) or (self in All Text near/6 management in All Text))

\# 38((patient in All Text near/6 education* in All Text) or (patient in All Text near/6 compliance in All Text) )

\# 39((health in All Text near/6 promotion* in All Text) or (health in All Text near/6 behavior* in All Text) or (health in All Text near/ 6 behaviour* in All Text))

\# 40(program in All Text near/6 evaluation* in All Text)

\# 41((behavioral in All Text near/6 intervention* in All Text) or (behavioral in All Text near/6 therap* in All Text) or (behavioral in All Text near/6 treatment* in All Text) or (behavioral in Abstract near/6 chang* in Abstract))

\# 42((behavioural in All Text near/6 treatment* in All Text) or (behavioural in All Text near/6 therap* in All Text) or (behavioural in All Text near/6 intervention* in All Text) or (behavioural in All Text near/6 chang* in All Text))

\# 43((psychosocial in All Text near/6 intervention* in All Text) or (psychosocial in All Text near/6 treatment* in All Text) or (psychosocial in All Text near/6 therap* in All Text))

\# 44 ((psycho-social in All Text near/6 intervention* in All Text) or (psycho-social in All Text near/6 treatment* in All Text) or (psychosocial in All Text near/6 therap* in All Text))

\# 45(adherence in All Text or (c in All Text and ompliance in All Text))

\# 46((lifestyle in All Text near/6 intervention* in All Text) or (lifestyle in All Text near/6 chang* in All Text))

\# 47((life-style in All Text near/6 intervention* in All Text) or (life-style in All Text near/6 chang* in All Text))

\# 48MeSH descriptor Weight loss explode all trees

\# 49((weight in All Text near/6 management in All Text) or (weight in All Text near/6 los* in All Text) or (weight in All Text near/6 reduction* in All Text))

$\# 50$ (\#29 or \#30 or \#31 or \#32 or \#33 or \#34 or \#35 or \#36 or \#37 or \#38 or \#39 or \#40 or \#41 or \#42 or \#43 or \#44 or \#45 or \# 46 or $\# 47$ or \#48 or \#49)

\# 51 (\#28 and \#50)

\section{MEDLINE}

1 exp Diabetes Mellitus, Type 2/

2 (MODY or NIDDM or T2DM or T2D).tw,ot.

3 (non insulin\$ depend\$ or noninsulin\$ depend\$ or noninsulin?depend\$ or non insulin?depend\$).tw,ot.

4 ((typ? 2 or typ? II or typ? 2 or typ?II) adj3 diabet $\$$ ).tw,ot.

5 ((late or adult $\$$ or matur $\$$ or slow or stabl\$) adj3 onset) and diabet\$).tw,ot.

6 or/1-5

7 exp Diabetes Insipidus/

8 diabet\$ insipidus.tw,ot.

97 or 8

106 not 9

11 exp Mental Disorders/

12 exp Psychotic disorders/

13 exp Mood disorders/

14 exp Personality disorders/

15 exp Bipolar disorders/

16 exp Schizophrenia/

17 (mental adj6 (disorder* or illness)).tw,ot.

18 (schizo* or psycho*).tw,ot.

19 ((bipolar or affective or personality) adj6 disorder*).tw,ot.

20 exp Depression/

21 depression*.tw,ot. 


\section{(Continued)}

22 or/11-21

2310 and 22

24 exp Patient Education as Topic/

25 exp Patient Compliance/

26 exp Self Carel

27 exp Health Promotion/

28 exp Behavior Therapy/

29 exp Health Behavior/

30 exp Program Evaluation/

31 exp Life style/

32 (self adj6 (care or management or monitoring)).tw,ot.

33 (patient adj6 (education* or compliance)).tw,ot.

34 (health adj6 (promotion* or behavio? $\left.{ }^{*}\right)$ ).tw,ot.

35 (program adj6 evaluation*).tw,ot.

36 (behavio?ral adj6 (intervention* or therap* or treatment* or chang*)).tw,ot.

37 ((psychosocial or psycho-social) adj6 (intervention* or treatment* ${ }^{*}$ or therap*)).tw,ot.

38 (adherence or compliance).mp.

39 ((lifestyle or life style) adj6 (intervention* or chang*)).tw,ot.

40 exp Weight Loss/

41 (weight adj6 (management or los* or reduction*)).tw,ot.

42 (lifestyle adj6 (intervention* or chang*)).tw,ot.

43 or/24-42

4423 and 43

45 randomized controlled trial.pt.

46 controlled clinical trial.pt.

47 randomi?ed.ab.

48 placebo.ab.

49 drug therapy.fs.

50 randomly.ab.

51 trial.ab.

52 groups.ab.

53 or/45-52

54 Meta-analysis.pt.

55 exp Technology Assessment, Biomedical/

56 exp Meta-analysis/

57 exp Meta-analysis as topic/

58 hta.tw,ot.

59 (health technology adj6 assessment\$).tw,ot.

60 (meta analy\$ or metaanaly\$ or meta?analy\$).tw,ot.

61 (search* adj10 (medical databas*or medline or pubmed or embase or cochrane or cinahl or psycinfo or psyclit or healthstar or biosis or current content*)).tw,ot.

62 (systematic adj3 review*).tw,ot.

63 or/54-62

6453 or 63

65 (comment or editorial or historical-article).pt.

6664 not 65

6744 and 66

\section{EMBASE}


1 exp Diabetes Mellitus, Type $2 /$

2 (MODY or NIDDM or T2D or T2DM).tw,ot.

3 ((typ? 2 or typ? II or typ?II or typ?2) adj3 diabet*).tw,ot.

4 (obes* adj3 diabet*).tw,ot.

5 (non insulin* depend* or non insulin?depend* or noninsulin* depend* or noninsulin?depend*).tw,ot.

6 ((adult* or matur* or late or slow or stabl*) adj3 diabet*).tw,ot.

7 or/1-6

8 exp diabetes insipidus/

9 diabet* insipidus.tw,ot.

108 or 9

117 not 10

12 exp mental disease/

13 exp psychosis/

14 exp mood disorder/

15 exp personality disorder/

16 exp bipolar disorder/

17 exp schizophrenia/

18 exp depression/

19 (mental adj6 (disorder* or disease* or illness)).tw,ot.

20 (schizo* or psycho*).tw,ot.

21 ((bipolar or affective or personality) adj6 (diseas* or disorder)).tw,ot.

22 depression*.tw,ot.

23 or/ $12-22$

2411 and 23

25 exp patient education/

26 exp patient compliance/

27 exp self care/

28 exp health promotion/

29 exp behavior therapy/

30 *health care quality/

31 exp lifestyle/

32 (self adj6 (care or management or monitoring)).tw,ot.

33 (patient adj6 (education* or compliance)).tw,ot.

34 (health adj6 (promotion* or behavio? $\left.{ }^{*}\right)$ ).tw,ot.

35 (program adj6 evaluation*).tw,ot.

36 (behavio?ral adj6 (intervention* or therap* or treatment* or chang*)).tw,ot.

37 ((psychosocial or psyco social) adj6 (intervention* or treatment* or therap*)).tw,ot.

38 (adherence or compliance).mp.

39 ((lifestyle or life style) adj6 (intervention* or chang*)).tw,ot.

40 exp weight reduction/

41 (weight adj6 (management or los* or reduction*)).tw,ot.

42 or $/ 25-41$

4324 and 42

44 exp Randomized Controlled Trial/

45 exp Controlled Clinical Trial/

46 exp Clinical Trial/

47 exp Comparative Study/

48 exp Drug comparison/ 


\section{(Continued)}

49 exp Randomization/

50 exp Crossover procedure/

51 exp Double blind procedure/

52 exp Single blind procedure/

53 exp Placebo/

54 exp Prospective Study/

55 ((clinical or control\$ or comparativ\$ or placebo\$ or prospectiv\$ or randomi?ed) adj3 (trial\$ or stud\$)).ab,ti.

56 (random\$ adj6 (allocat\$ or assign $\$$ or basis or order\$)).ab,ti.

57 ((singl\$ or doubl\$ or trebl\$ or tripl\$) adj6 (blind\$ or mask\$)).ab,ti.

58 (cross over or crossover).ab,ti.

59 or/44-58

60 exp meta analysis/

61 (metaanaly\$ or meta analy\$ or meta?analy\$).ab,ti,ot.

62 (search\$ adj10 (medical database\$ or medline or pubmed or embase or cochrane or cinahl or psycinfo or psyclit or healthstar or biosis or current content $\$$ or systematic $\$)$ ).ab,ti,ot.

63 (review* $^{*}$ adj6 systematic).tw,ot.

64 exp Literature/

65 exp Biomedical Technology Assessment/

66 hta.tw,ot.

67 (health technology adj6 assessment\$).tw,ot.

68 or/60-67

6959 or 68

70 (comment or editorial or historical-article).pt.

7169 not 70

7243 and 71

\section{PsycINFO}

1 (MODY or NIDDM or T2DM or T2D).tw,ot.

2 (non insulin\$ depend\$ or noninsulin\$ depend\$ or noninsulin?depend\$ or non insulin?depend\$).tw,ot.

3 ((typ? 2 or typ? II or typ?2 or typ?II) adj3 diabet\$).tw,ot.

4 (( (late or adult $\$$ or matur\$ or slow or stabl\$) adj3 onset) and diabet $\$)$.tw,ot.

5 or/ $1-4$

6 exp Diabetes Insipidus/

7 diabet $\$$ insipidus.tw,ot.

86 or 7

95 not 8

10 exp Mental Disorders/

11 exp Mood disorders/

12 exp Personality disorders/

13 exp Schizophrenia/

14 (mental adj6 (disorder* or illness)).tw,ot.

15 (schizo* or psycho*).tw,ot.

16 ((bipolar or affective or personality) adj6 (disorder* or illness)).tw,ot.

17 exp Depression/

18 depression*.tw,ot.

19 or/ $10-18$

209 and 19

21 exp Self Care/ 


\section{(Continued)}

22 exp Health Promotion/

23 exp Behavior Therapy/

24 exp Health Behavior/

25 exp Program Evaluation/

26 (self adj6 (care or management or monitoring)).tw,ot.

27 (patient adj6 (education* or compliance)).tw,ot.

28 (health adj6 (promotion* or behavio? $\left.{ }^{*}\right)$ ).tw,ot.

29 (program adj6 evaluation*).tw,ot.

30 (behavio?ral adj6 (intervention* or therap* or treatment* or chang*)).tw,ot.

31 ((psychosocial or psycho-social) adj6 (intervention* or treatment* ${ }^{*}$ or therap*)).tw,ot.

32 (adherence or compliance).mp.

33 ((lifestyle or life style) adj6 (intervention* or chang*)).tw,ot.

34 exp Weight Loss/

35 (weight adj6 (management or los* or reduction*)).tw,ot.

36 or/21-35

3720 and 36

38 randomi?ed.ab.

39 placebo.ab.

40 randomly.ab.

41 trial.ab.

42 groups.ab.

43 or/38-42

44 exp Meta-analysis/

45 hta.tw,ot.

46 (health technology adj6 assessment\$).tw,ot.

47 (meta analy\$ or metaanaly $\$$ or meta?analy\$).tw,ot.

48 (search* adj10 (medical databas*or medline or pubmed or embase or cochrane or cinahl or psycinfo or psyclit or healthstar or biosis or current content $\left.t^{*}\right)$.tw,ot.

49 (systematic adj3 review*).tw,ot.

50 or/44-49

5143 or 50

5237 and 51

\section{AMED}

1. exp diabetes mellitus type $2 /$

2. (MODY or NIDDM or T2DM or T2D).mp. [mp=abstract, heading words, title]

3. (non insulin $\$$ depend $\$$ or noninsulin $\$$ depend $\$$ or noninsulin?depend $\$$ or non insulin?depend \$).mp. [mp=abstract, heading words, title]

4. ((typ? 2 or typ? II or typ?2 or typ?II) adj3 diabet $\$$ ).mp. [mp=abstract, heading words, title]

5. ((late or adult $\$$ or matur $\$$ or slow or stabl\$) adj3 onset) and diabet $\$) . \mathrm{mp}$. [mp=abstract, heading words, title]

6. 1 or 2 or 3 or 4 or 5

7. exp Mental disorders/

8. (mental adj6 (disorder* or illness)).mp. [mp=abstract, heading words, title]

9. (schizo* or psycho*).mp. [mp=abstract, heading words, title]

10. ((bipolar or affective or personality) adj6 disorder*).mp. [mp=abstract, heading words, title]

11. exp depression/

12. depression*.mp. [mp=abstract, heading words, title]

13. 7 or 8 or 9 or 10 or 11 or 12

14.6 and 13

Self-management interventions for type 2 diabetes in adult people with severe mental illness (Protocol)

Copyright @ 2014 The Cochrane Collaboration. Published by John Wiley \& Sons, Ltd. 


\section{(Continued)}

15. exp patient education/

16. exp self care/

17. health education/

18. exp health promotion/

19. exp health behavior/

20. exp program evaluation/

21. exp life style/

22. (self adj6 (care or management or monitoring)).mp. [mp=abstract, heading words, title]

23. (patient adj6 (education* or compliance)).mp. [mp=abstract, heading words, title]

24. (health adj6 (promotion* or behavio? $\left.{ }^{*}\right)$ ).mp. [mp=abstract, heading words, title]

25. (program adj6 evaluation*).mp. [mp=abstract, heading words, title]

26. (behavio?ral adj6 (intervention* or therap* or treatment* or chang*)).mp. [mp=abstract, heading words, title]

27. ((psychosocial or psycho-social) adj6 (intervention* or treatment* or therap*)).mp. [mp=abstract, heading words, title]

28. (adherence or compliance).mp. [mp=abstract, heading words, title]

29. ((lifestyle or life style) adj6 (intervention* or chang*)).mp. [mp=abstract, heading words, title]

30. exp Weight loss/

31. (weight adj6 (management or los* or reduction*)).mp. [mp=abstract, heading words, title]

32. (lifestyle adj6 (intervention* or chang*)).mp. [mp=abstract, heading words, title]

33.15 or 16 or 17 or 18 or 19 or 20 or 21 or 22 or 23 or 24 or 25 or 26 or 27 or 28 or 29 or 30 or 31 or 32

34.14 and 33

35. exp clinical trials/

36. exp comparative study/

37. exp random allocation/

38. exp Placebos/

39. exp prospective studies/

40. ( (clinical or control\$ or comparativ\$ or placebo $\$$ or prospectiv\$ or randomi?ed) adj3 (trial\$ or stud $\$)$ ).mp. [mp=abstract, heading words, title]

41. (random $\$$ adj6 (allocat $\$$ or assign $\$$ or basis or order $\$)$ ).mp. [mp=abstract, heading words, title]

42. ((singl\$ or doubl\$ or trebl\$ or tripl\$) adj6 (blind $\$$ or mask\$)).mp. [mp=abstract, heading words, title]

43. (cross over or crossover).mp. [ $\mathrm{mp}=\mathrm{abstract}$, heading words, title]

44.35 or 36 or 37 or 38 or 39 or 40 or 41 or 42 or 43

45. exp Meta analysis/

46. (metaanaly $\$$ or meta analy $\$$ or meta?analy $\$$ ).mp. [mp=abstract, heading words, title]

47. (review* adj6 systematic).mp. [mp=abstract, heading words, title]

48. (search* adj10 (medical databas*or medline or pubmed or embase or cochrane or cinahl or psycinfo or psyclit or healthstar or biosis or current content $\left.t^{*}\right)$.mp. [mp=abstract, heading words, title]

49. hta.mp. [mp=abstract, heading words, title]

50. (health technology adj6 assessment $\$$ ).mp. [mp=abstract, heading words, title]

51.45 or 46 or 47 or 48 or 49 or 50

52. 44 or 51

53. 34 and 52

\section{HTA}

1. exp diabetes mellitus type $2 /$

2. (MODY or NIDDM or T2DM or T2D).mp. [mp=title, text, subject heading word]

3. (non insulin\$ depend $\$$ or noninsulin $\$$ depend $\$$ or noninsulin?depend $\$$ or non insulin?depend $\$$ ). mp. [mp=title, text, subject heading word]

4. ((typ? 2 or typ? II or typ?2 or typ?II) adj3 diabet\$).mp. [mp=title, text, subject heading word]

5. (((late or adult\$ or matur\$ or slow or stabl\$) adj3 onset) and diabet\$).mp. [mp=title, text, subject heading word]

Self-management interventions for type 2 diabetes in adult people with severe mental illness (Protocol)

Copyright @ 2014 The Cochrane Collaboration. Published by John Wiley \& Sons, Ltd. 


\section{(Continued)}

6. 1 or 2 or 3 or 4 or 5

7. exp Mental disorders/

8. (mental adj6 (disorder* or illness)).mp. [mp=title, text, subject heading word]

9. (schizo* or psycho*).mp. [mp=title, text, subject heading word]

10. ((bipolar or affective or personality) adj6 disorder $\left.{ }^{*}\right) . \mathrm{mp}$. [mp=title, text, subject heading word]

11. exp depression/

12. depression*.mp. [mp=title, text, subject heading word]

13. 7 or 8 or 9 or 10 or 11 or 12

14.6 and 13

15. exp self care/

16. health education/

17. exp health promotion/

18. exp health behavior/

19. exp program evaluation/

20. exp life style/

21. (self adj6 (care or management or monitoring)).mp. [mp=title, text, subject heading word]

22. (patient adj6 (education* or compliance)).mp. [mp=title, text, subject heading word]

23. (health adj6 (promotion* or behavio? $\left.{ }^{*}\right)$ ).mp. [mp=title, text, subject heading word]

24. (program adj6 evaluation*).mp. [mp=title, text, subject heading word]

25. (behavio?ral adj6 (intervention* or therap* or treatment* or chang*)).mp. [mp=title, text, subject heading word]

26. ((psychosocial or psycho-social) adj6 (intervention* or treatment* or therap* $\left.{ }^{*}\right)$.mp. $[\mathrm{mp}=$ title, text, subject heading word]

27. (adherence or compliance).mp. [mp=title, text, subject heading word]

28. ((lifestyle or life style) adj6 (intervention* or chang*)).mp. [mp=title, text, subject heading word]

29. exp Weight loss/

30. (weight adj6 (management or los* or reduction*)).mp. [mp=title, text, subject heading word]

31. (lifestyle adj6 (intervention* or chang*)).mp. [mp=title, text, subject heading word]

32. exp comparative study/

33. exp random allocation/

34. exp Placebos/

35. exp prospective studies/

36. ( (clinical or control $\$$ or comparativ $\$$ or placebo $\$$ or prospectiv $\$$ or randomi?ed) adj3 (trial $\$$ or stud $\$)$ ). mp. $[\mathrm{mp}=$ title, text, subject heading word]

37. (random $\$$ adj6 (allocat $\$$ or assign $\$$ or basis or order $\$)$ ).mp. [mp=title, text, subject heading word]

38. ((singl\$ or doubl\$ or trebl\$ or tripl\$) adj6 (blind $\$$ or mask\$)).mp. [mp=title, text, subject heading word]

39. (cross over or crossover).mp. [mp=title, text, subject heading word]

40. exp Meta analysis/

41. (metaanaly\$ or meta analy\$ or meta?analy\$).mp. [mp=title, text, subject heading word]

42. (review* adj6 systematic).mp. [mp=title, text, subject heading word]

43. (search* adj10 (medical databas*or medline or pubmed or embase or cochrane or cinahl or psycinfo or psyclit or healthstar or biosis or current content $\left.\mathrm{t}^{*}\right)$.mp. [mp=title, text, subject heading word]

44.15 or 16 or 17 or 18 or 19 or 20 or 21 or 22 or 23 or 24 or 25 or 26 or 27 or 28 or 29 or 30 or 31

45.32 or 33 or 34 or 35 or 36 or 37 or 38 or 39

46. 40 or 41 or 42 or 43

47. 45 or 46

48. 14 and 44 and 47

\section{NHS EDD}


1. exp diabetes mellitus type $2 /$

2. (MODY or NIDDM or T2DM or T2D).mp. [mp=title, text, subject heading word]

3. (non insulin\$ depend\$ or noninsulin\$ depend\$ or noninsulin?depend\$ or non insulin?depend\$).mp. [mp=title, text, subject heading word]

4. ((typ? 2 or typ? II or typ?2 or typ?II) adj3 diabet\$).mp. [mp=title, text, subject heading word]

5. ((late or adult $\$$ or matur $\$$ or slow or stabl\$) adj3 onset) and diabet\$).mp. [mp=title, text, subject heading word]

6. 1 or 2 or 3 or 4 or 5

7. exp Mental disorders/

8. (mental adj6 (disorder* or illness)).mp. [mp=title, text, subject heading word]

9. (schizo* or psycho*).mp. [mp=title, text, subject heading word]

10. ((bipolar or affective or personality) adj6 disorder*).mp. [mp=title, text, subject heading word]

11. exp depression/

12. depression*.mp. [mp=title, text, subject heading word]

13. 7 or 8 or 9 or 10 or 11 or 12

14. 6 and 13

15. exp patient education/

16. exp self care/

17. health education/

18. exp health promotion/

19. exp health behavior/

20. exp program evaluation/

21. exp life style/

22. (self adj6 (care or management or monitoring)).mp. [mp=title, text, subject heading word]

23. (patient adj6 (education* or compliance)).mp. [mp=title, text, subject heading word]

24. (health adj6 (promotion* or behavio? $\left.\mathrm{r}^{*}\right)$ ).mp. [mp=title, text, subject heading word]

25. (program adj6 evaluation*).mp. [mp=title, text, subject heading word]

26. (behavio?ral adj6 (intervention* or therap* or treatment* or chang*)).mp. [mp=title, text, subject heading word]

27. ((psychosocial or psycho-social) adj6 (intervention* or treatment* or therap*)).mp. [mp=title, text, subject heading word]

28. (adherence or compliance).mp. [mp=title, text, subject heading word]

29. ((lifestyle or life style) adj6 (intervention* or chang*)).mp. [mp=title, text, subject heading word]

30. exp Weight loss/

31. (weight adj6 (management or los* or reduction*)).mp. [mp=title, text, subject heading word]

32. (lifestyle adj6 (intervention* ${ }^{*}$ or chang*)).mp. [mp=title, text, subject heading word]

33.15 or 16 or 17 or 18 or 19 or 20 or 21 or 22 or 23 or 24 or 25 or 26 or 27 or 28 or 29 or 30 or 31 or 32

34. 14 and 33

35. exp comparative study/

36. exp random allocation/

37. exp Placebos/

38. exp prospective studies/

39. ( (clinical or control $\$$ or comparativ $\$$ or placebo $\$$ or prospectiv $\$$ or randomi?ed) adj3 (trial $\$$ or stud $\$)$ ).mp. [mp=title, text, subject heading word]

40. (random $\$$ adj6 (allocat $\$$ or assign $\$$ or basis or order $\$)$ ).mp. [mp=title, text, subject heading word]

41. ((singl\$ or doubl\$ or trebl\$ or tripl\$) adj6 (blind $\$$ or mask $\$)$ ).mp. [mp=title, text, subject heading word]

42. (cross over or crossover).mp. [mp=title, text, subject heading word]

43. exp Meta analysis/

44. (metaanaly\$ or meta analy\$ or meta?analy\$).mp. [mp=title, text, subject heading word]

45. (review* adj6 systematic).mp. [mp=title, text, subject heading word]

46. (search* adj10 (medical databas*or medline or pubmed or embase or cochrane or cinahl or psycinfo or psyclit or healthstar or

Self-management interventions for type 2 diabetes in adult people with severe mental illness (Protocol) 


\section{(Continued)}

biosis or current content*)).mp. [mp=title, text, subject heading word]

47. hta.mp. [mp=title, text, subject heading word]

48. (health technology adj6 assessment $\$$ ).mp. [mp=title, text, subject heading word]

49. 43 or 44 or 45 or 46 or 47 or 48

50.35 or 36 or 37 or 38 or 39 or 40 or 41 or 42

51.49 or 50

52.34 and 51

\section{CINAHL Plus}

S1 (MH "Diabetes Mellitus, Type 2")

S2 (MODY or NIDDM or T2DM or T2D)

S3 (non insulin* depend* or noninsulin* depend* or noninsulin?depend* or non insulin?depend*)

S4 ((typ* 2 or typ* II or typ\#2 or typ\#II) N3 diabet $\left.^{*}\right)$

S5 (((late or adult* or matur* or slow or stabl*) N3 onset) and diabet*)

S6 S1 or S2 or S3 or S4 or S5

S7 (MH "Mental Disorders, Chronic") OR (MH “Mental Disorders+") OR (MH "Psychotic Disorders+") OR (MH "Personality Disorders+”) OR (MH “Depression+")

S8 (MH "Bipolar Disorder+")

S9 (mental N6 (disorder* or disease* or illness))

S10 (schizo* or psycho*)

S11 ((bipolar or affective or personality) N6 (diseas* or disorder))

S12 depression*

S13 S7 or S8 or S9 or S10 or S11 or S12

S14 (MH "Patient Education") OR (MH "Health Education") OR (MH “Diabetes Education")

S15 (MH "Self Care") OR (MH "Self Administration")

S16 (MH "Health Behavior+")

S17 (MH "Program Evaluation")

S18 (MH "Behavior Therapy")

S19 (MH "Life Style") OR (MH "Life Style, Sedentary") OR (MH "Life Style Changes")

S20 (MH "Health Promotion")

S21 (self N6 (care or management or monitoring))

S22 (patient N6 (education* or compliance))

S23 (health N6 (promotion* or behavio\# $\left.{ }^{*}\right)$ )

S24 (program N6 evaluation*)

S25 (behavio\#ral N6 (intervention* or therap* or treatment* or chang*))

S26 ((psychosocial or psycho-social) N6 (intervention* or treatment* or therap*))

S27 (adherence or compliance)

S28 ((lifestyle or life style) N6 (intervention* or chang*))

S29 (MH "Weight Loss")

S30 (MH "Weight Reduction Programs")

S31 (weight N6 (management or los* or reduction*))

S32 (lifestyle N6 (intervention* or chang*))

S33 S14 or S15 or S16 or S17 or S18 or S19 or S20 or S21 or S22 or S23 or S24 or S25 or S26 or S27 or S28 or S29 or S30 or S31 or $\mathrm{S} 32$

S34 (MH "Clinical Trials+”)

S35 (MH "Comparative Studies")

S36 (MH "Random Assignment")

S37 (MH "Prospective Studies") 


\section{(Continued)}

S38 ((clinical or control* or comparativ* or placebo* or prospectiv* or randomi\#ed) N3 (trial* or stud $\left.{ }^{*}\right)$ )

S39 (random* N6 (allocat* or assign* or basis or order*))

S40 ((singl* or doubl* or trebl* or tripl*) N6 (blind* or mask*))

S41 (cross over or crossover)

S42 S34 or S35 or S36 or S37 or S38 or S39 or S40 or S41

S43 (MH "Meta Analysis")

S44 (metaanaly* or meta analy* or meta\#analy*)

S45 (review* N6 systematic)

S46 (search* N10 (medical database* or medline or pubmed or embase or cochrane or cinahl or psycinfo or psyclit or healthstar or biosis or current content* or systematic*))

S47 (MH "Literature Review+")

S48 hta

S49 (health technology N6 assessment*)

S50 S43 or S44 or S45 or S46 or S47 or S48 or S49

$\mathrm{S} 51 \mathrm{~S} 42$ or $\mathrm{S} 50$

S52 S6 AND S13 AND S33 AND S51

\section{CONTRIBUTIONSOFAUTHORS}

Hayley McBain (HM): protocol draft, search strategy development, acquiring trial reports, trial selection, data extraction, data analysis, data interpretation, review draft and future review updates.

Kathleen Mulligan (KM): protocol draft, search strategy development, data extraction, data analysis, data interpretation, review draft and future review updates.

Mark Haddad (MH): protocol draft, trial selection, data analysis, data interpretation, review draft and future review updates.

Chris Flood (CF): protocol draft, trial selection, data analysis, data interpretation, review draft and future review updates.

Julia Jones $(\mathrm{JJ})$ : protocol draft, data analysis, data interpretation, review draft and future review updates.

Alan Simpson (AS): protocol draft, data extraction, data analysis, data interpretation, review draft and future review updates

\section{DECLARATIONSOF INTEREST}

HM: None known.

KM: None known.

MH: None known.

CF: None known.

JJ: None known.

AS: None known. 


\section{NOTES}

The methods section and appendix 1 of this protocol are based on a standard template established by the Cochrane Metabolic and Endocrine Disorders Group. 